\title{
Optimization of Consignment-Store-Based Supply Chain with Black Hole Algorithm
}

\author{
Ágota Bányai, Tamás Bányai, and Béla Illés \\ University of Miskolc, Miskolc, Hungary \\ Correspondence should be addressed to Tamás Bányai; alttamas@uni-miskolc.hu
}

Received 23 February 2017; Revised 24 May 2017; Accepted 15 August 2017; Published 27 September 2017

Academic Editor: Petri T. Helo

Copyright (C) 2017 Ágota Bányai et al. This is an open access article distributed under the Creative Commons Attribution License, which permits unrestricted use, distribution, and reproduction in any medium, provided the original work is properly cited.

The globalization of economy and market led to increased networking in the field of manufacturing and services. These manufacturing and service processes including supply chain became more and more complex. The supply chain includes in many cases consignment stores. The design and operation of these complex supply chain processes can be described as NP-hard optimization problems. These problems can be solved using sophisticated models and methods based on metaheuristic algorithms. This research proposes an integrated supply model based on consignment stores. After a careful literature review, this paper introduces a mathematical model to formulate the problem of consignment-store-based supply chain optimization. The integrated model includes facility location and assignment problems to be solved. Next, an enhanced black hole algorithm dealing with multiobjective supply chain model is presented. The sensitivity analysis of the heuristic black hole optimization method is also described to check the efficiency of new operators to increase the convergence of the algorithm. Numerical results with different datasets demonstrate how the proposed model supports the efficiency, flexibility, and reliability of the consignment-store-based supply chain.

\section{Introduction}

In today's economy, the pressure is on to make the operations of supply chain from purchasing to distribution more and more efficient. The competition is characterized as competition between supply chain networks rather than competition between individual production and service companies. The upstream and downstream linkages of involved organizations like production and service companies, suppliers, 3PL or 4PL providers, wholesalers, and retailers increase the complexity of supply chain networks. This increased complexity led to the implementation of new strategies and tools to make supply chain structure more transparent, while the efficiency and flexibility are increased. One of these tools is the consignment inventory concept. Consignment inventory gives advantages for both the suppliers and the customers since the supplier enjoys the advantages of close connection with customers, decrease of own store capacity, and decreased transportation and packaging costs. Meanwhile, customers using consignment-inventory-based supply can enjoy advantages provided by consignment inventory like low supply risk, decreased supply costs, and transparency of inventories.

The design and operation of consignment inventory or consignment-store-based supply chains include a huge number of problems: facility location, routing, scheduling, budgeting, transportation problem, inventory optimization, assignment, and queuing problems.

The model presented in this work not only combines the facility location of consignment stores and the assignment problems of stores, suppliers, and customers but also takes into account capacity of logistic resources. To the best of our knowledge, the facility location of consignment stores in supply chains and its assignment to customers and suppliers has not been considered in the current literature.

The main contributions of this work include (1) an integrated consignment-store-based supply chain model that combines facility location planning and assignment of involved organizations of the supply chain, (2) a black-holeoptimization-based algorithm, which includes new heuristic operators to increase the convergence, (3) a test of the 
TABLE 1: Selected papers related to topics of the research.

\begin{tabular}{|c|c|c|c|}
\hline SN & Author(s) & Year & Topics \\
\hline 1 & Hallikas and Lintukangas & 2016 & Risk management in purchasing and supply chain \\
\hline 2 & Matopoulos et al. & 2016 & Modelling in purchasing and supply chain \\
\hline 3 & Immonen et al. & 2016 & Supply chain in B2B services \\
\hline 4 & Zhang et al. & 2017 & In-plant supply chain design, production routing \\
\hline 5 & Govindan and Soleimani & 2017 & Supply chain management in reverse logistics \\
\hline 6 & Ma et al. & 2016 & Integrated supply chain design \\
\hline 7 & Diabat and Deskoores & 2016 & Supply chain optimization with hybrid genetic algorithm \\
\hline 8 & Pishvaee and Rabbani & 2011 & Responsive supply chain network design with heuristics \\
\hline 9 & Liu and Chen & 2011 & Inventory routing in a supply chain with heuristics \\
\hline 10 & Chávez et al. & 2017 & Simulation-based supply chain modelling \\
\hline 11 & Dorigatti et al. & 2016 & Agent-based simulation of collaborative supply chain \\
\hline 12 & Ge et al. & 2016 & Simulation and hybrid optimization of supply chain \\
\hline 13 & Ben Othman et al. & 2017 & Resource scheduling with decision support system \\
\hline 14 & Dey et al. & 2017 & Facility location in supply chain \\
\hline 15 & Zahran et al. & 2016 & Consignment stock modelling with delay-in-payment \\
\hline 16 & Hackett & 1993 & Consignment contracting \\
\hline 17 & Bylka & 2013 & Noncooperative consignment stock strategies \\
\hline 18 & Bazan et al. & 2014 & Consignment stock agreements for two-level supply chain \\
\hline 19 & Li et al. & 2014 & Supply diversification \\
\hline 20 & Batarfi et al. & 2016 & Strategy for dual-channel supply chain \\
\hline 21 & Ru and Wang & 2010 & Consignment contracting \\
\hline 22 & Fraser & 2016 & Schwarzschild radius \\
\hline 23 & Piotrowski et al. & 2014 & Black hole optimization versus other heuristics \\
\hline 24 & Dorigo and Gambardella & 1997 & Ant colony optimization \\
\hline 25 & Yang & 2014 & Nature-inspired optimization algorithms \\
\hline 26 & Bhargava et al. & 2013 & Cuckoo search optimization \\
\hline 27 & Lozano et al. & 2017 & Artificial bee colony algorithm \\
\hline 28 & Niknam et al. & 2013 & Bat-inspired heuristics \\
\hline 29 & McKendall Jr. et al. & 2006 & Simulated annealing heuristics \\
\hline 30 & Saha et al. & 2014 & Gravitation search algorithm \\
\hline 31 & Srivastava & 2015 & Intelligent water drop optimization \\
\hline 32 & Bányai et al. & 2015 & Harmony search optimization \\
\hline 33 & Zhang et al. & 2008 & Random black hole particle swarm optimization \\
\hline 34 & Yaghoobi and Mojallali & 2016 & Black hole algorithm with genetic operators \\
\hline 35 & Wang et al. & 2016 & Black hole base optimization \\
\hline 36 & Bouchekara & 2013 & Black-hole-based optimization technique \\
\hline 37 & Wang et al. & 2014 & Parameter optimization based on black hole algorithm \\
\hline 38 & Hatamlou & 2013 & Data clustering with black hole algorithm \\
\hline 39 & Azizipanah-Abarghooee et al. & 2014 & Power system scheduling with black hole optimization \\
\hline 40 & Bouchekara & 2014 & Power flow optimization with black hole algorithm \\
\hline
\end{tabular}

modified black hole algorithm with different datasets and test functions based on CEC 2005, and (4) computational results of consignment-store-based supply chain problems with different datasets.

This paper is organized as follows. Section 2 presents a literature review, which systematically summarizes the research background of supply chain, consignment stores, and black hole optimization. Section 3 describes the model framework of the consignment-store-based supply chains. Section 4 presents the black hole optimization and supposes some modification to improve its convergence and enhance its efficiency. Section 5 demonstrates the sensitivity analysis of the algorithm based on CEC 2005 functions. For our study, in Section 6, we focus on the optimization results with numerical analysis. Conclusions and future research directions are discussed in Section 7.

\section{Literature Review}

Since our study embraces several related research streams, namely, supply chain management, consignment stores, and black hole optimization, we provide a brief review on each stream before to elaborate the model, algorithm, and solution. Table 1 enlists the papers published in these areas related to our research.

Only limited attention has been paid to consignmentstore-based supply chain optimization with metaheuristic methods in the literature. There has recently been an 
increased interest in performance analysis of supply chains [1-14] and some recent analysis has been targeted specifically towards the integration of consignment stores into supply chain processes [15-21] and optimization with metaheuristics based on swarming algorithms [22-40], especially black hole optimization. Firstly, the relevant terms were defined and the initial searches were conducted. The keywords used in the search were supply chain management, supply, logistics, consignment store, consignment contract, heuristics, metaheuristics, and black hole optimization. The literature sources were found through scientific databases (ScienceDirect, Scopus, and Web of Science) and regular search engines (Google) and included journal articles. Initially, more than 300 articles were identified. This list was narrowed down to 42 titles by selecting journal articles focusing on our research field. It is worth mentioning that the search was conducted in February 2017; therefore new articles may have been published since then.

2.1. Research on Supply Chain Management. Successful production and service processes provide a significant competitive advantage over other participants of the market. Logistics-related operations such as warehousing, transportation, loading unit building, packaging, customer service, and inventory carrying account for up to $95 \%$ of the total cost, depending on the corporate sector. For that reason, it is important to take the logistics-related operations and processes under control and turn logistics into the source of competitive edges. Logistics can be divided into four important parts: purchasing, production or service, distribution, and recycling.

Supply chain management influences the efficiency of purchasing, because supplier orientation, supplier dependency, customer orientation, and purchasing strategy have an effect on performance [1]. Supply chain modelling in purchasing is important not just at the functional and operational level but also at the organizational and strategic level [2]. The relationship between purchasing strategies and e-business solutions like business-to-business services has been studied in the literature and provides new knowledge on complex purchasing systems [3]. In-plant supply chain design includes the problems of facility location, production routing, and scheduling [4].

There is a growing interest in the field of closed-loop supply chain design and green supply chain. Reverse logistic systems play an important role in the end-of-life product recycling and influence consumer's return practices for collecting used products, like wastes of electric and electronic equipment [5]. The functional sequences of supply chain can be taken into consideration as an integrated process; the integration of production and distribution planning is a good solution to avoid conflict in sales [6].

The increased complexity of industrial and service processes led to the application of complex solution methods and procedures to optimize the parameters of related supply chains. Heuristic optimization methods are used to solve NPhard problems of supply chains: integrated supply chain problems can be solved by hybrid algorithms [7], graph-theorybased heuristic supports responsive supply chain design [8], and inventory routing and pricing problems in a supply chain can be solved with Tabu Search [9]. Simulation-based methods support the optimization of deterministic and stochastic models in the field of transportation [10], collaboration analysis for jointly working members [11], and supply chain optimization to find the core parameters of supply chain strategies to ensure cost efficiency [12]. Decision support systems make it possible to find optimal solutions for resource scheduling in supply chain [13] and group decision-making is an effective tool for facility location problems of supply chains [14].

2.2. Research on Consignment Stores. There is a great body of research dealing with consignment policies, consignment stores, and consignment strategies. Within the frame of this chapter, we give a short overview on this reach literature source related to our research. Production companies usually have four types of inventories: raw materials, workin-process, finished products, and manufacturing supplies. Holding inventories makes it possible to avoid losses of sales, gain quantity discounts, reduce order costs, and achieve efficient production run. Consignment is a special coordination mechanism of inventories, because the owner keeps ownership of his goods and products until they are sold. Consignment stock can improve the supply chain improvement, because vendor uses its buyer's warehouse capacity to store goods. The operation of consignment-store-based supply chain depends on the policy of its operation; therefore, it is important to optimize its operation strategy [15]. The first work investigated the consignment contracting and defined the role of consignment; it can limit the middleman's commitment and increase the profitability of sales [16]. In a later study, the importance of noncooperative stock strategies was underlined and generalized consignment policies were considered to minimize the average total costs by individual decisions [17]. Another topic that has received significant attention in the literature is the analysis of multilevel supply chain with consignment stores. The result of these researches showed the following advantages of using consignment stores in supply chain [18]: improved customer service through decreased reaction time on customer's demands, levelling of customer's demands, decreased inventory on the side of the supplier [19], and cost reduction [20]. The possible control processes of consignment inventory have been studied in the literature; consignment arrangements from the point of view of suppliers and retailers are discussed [21].

2.3. Research on Black Hole Optimization. Black holes were predicted by Einstein's theory of general relativity. If the mass of a dead star's core is more than three times the solar mass, the force of gravity overwhelms all other forces. In a black hole, gravity pulls so much that nothing, not even particles, light, or radiation, can escape from it. The boundary of a black hole is called event horizon, beyond which events cannot be observed and particles cannot move in any direction but only closer to the core of the black hole. This boundary is called Schwarzschild radius, which is given as

$$
r_{s}=\frac{2 g M}{c^{2}},
$$


where $g$ is the gravitational constant, $M$ is the object mass, and $c$ is the speed of light [22].

The black hole optimization (BHO) is based on this phenomenon. Black hole optimization can be described as simplification of the well-known particle swarm optimization using inertia weight. The black hole optimization belongs to heuristics inspired by the laws of nature, like Newton's three laws of motion, his law of gravitation, the ideal gas law, and so on. The laws of nature may be as relevant sources of inspiration for heuristics as living bodies or humandepending phenomenon [23]. Living-bodies-inspired heuristics are, for example, ant colony optimization [24], firefly optimization [25], cuckoo search [26], artificial bee colony optimization [27], bat algorithm [28], or bacterial algorithm. Simulated annealing [29], gravitation search [30], intelligent water drops [31], and black hole algorithms [23] are inspired by physical laws, while harmony search [32] is based on a strongly human-depending attitude.

$\mathrm{BHO}$ can be used for hybrid metaheuristics [33]. Genetic operators can increase the convergence and the optimization results of $\mathrm{BHO}$ performing a more diverse search in the search space [34] and the convergence of the basic algorithm can be increased by improved measurement of distances in the search space [35]. The technique can be proposed for the optimization of different scientific problems, like pole face optimization of a magnetizer [36], optimization of parameters of least squares support vector machine [37], data clustering [38], scheduling of thermal power systems [39], or power flow optimization [40].

2.4. Analysis of Recent Papers. More than $80 \%$ of the articles were published in the last 4 years. This result indicates the scientific potential of this research field including the problems of supply chain, consignment stores, and heuristic optimization. The articles that addressed the optimization of supply chain processes are focusing on conventional manufacturing and service processes and only a few of them aimed to identify the optimization aspects of consignment-storebased supply chain. Therefore, the heuristic optimization of consignment-store-based supply still needs more attention and research, especially in the case of robust, networking cases. It was found that heuristic algorithms are important support tools for design, since a wide range of models determines an NP-hard optimization problem. According to that, the main focus of this research is on the modelling and optimization of consignment-store-based supply chain.

The aim of this paper is to investigate the effect of location of consignment stores on the performance of the whole supply chain. The contribution of this paper to the literature is twofold: description of a consignment-store-based supply chain model including the optimization problem of facility location and assignment and development of a black-holebased algorithm to solve an integrated optimization problem.

\section{Model Framework}

The model framework of the consignment-store-based supply chain is a two-level supply chain including suppliers, consignment stores, and customers (Figure 1). The supply chain has $m$ suppliers that produce the needs of $p$ different customers. The supplier and the customer want to set up a consignment store network to support the just-in-time and just-in-sequence supply. Depending on the number and location of consignment stores and consignment agreements, the suppliers are able to ship their products to different consignment stores and the customers are able to buy their needs from different consignment stores. The decision variables of this model are the following: optimal location of the consignment stores, types of consignment agreements between suppliers and customers, and assignment of objects of supply chain and order quantities. These decision variables include an integrated optimization problem: facility location problem and assignment problem.

The decision variables describe the decisions to be made. In this model, the following must be decided: (a) how many products from suppliers through consignment stores to customers should be transported; (b) location of each consignment store. These two decisions represent the abovementioned assignment and facility location problem. With this in mind, we define $q_{i j k}$ as amount of products transported from $i$ th suppliers through $j$ th consignment store to the $k$ th customer and $\left(x_{i}^{W}, y_{i}^{W}\right)$ as coordinates of the $j$ th consignment store.

The objective function of the problem describes the minimization of the costs of both the suppliers and the customers.

$$
\min C=\sum_{i=1}^{m} C_{i}^{S}+\sum_{k=1}^{p} C_{k}^{C},
$$

where $C_{i}^{S}$ represents the costs of suppliers and $C_{k}^{C}$ represents the costs of customers.

The first part of the cost function (2) includes the sum of transportation costs among suppliers and consignment stores, the warehousing costs, and the manufacturing costs of all suppliers.

$$
C_{i}^{S}=\sum_{j=1}^{n} \sum_{k=1}^{p} q_{i j k}\left[c_{j}^{W}+c_{i j}^{T} l_{i j}\left(x_{j}^{W}, y_{j}^{W}\right)\right]+C_{i}^{M},
$$

where $c_{j}^{W}$ is the specific warehousing cost in the $j$ th consignment store, $c_{i j}^{T}$ is the specific transportation cost between the $i$ th supplier and $j$ th consignment store, $l_{i j}$ is the length of the transportation route between the $i$ th supplier and $j$ th consignment store, $x_{j}^{W}$ and $y_{j}^{W}$ are the coordinates of the $j$ th consignment store, and $C_{i}^{M}$ is the manufacturing cost of the $i$ th supplier.

The second part of the cost function (2) includes the transportation costs from consignment stores to customers and the purchasing costs of products.

$$
C_{k}^{C}=\sum_{i=1}^{m} \sum_{j=1}^{n} q_{i j k}\left(c_{i}^{P}+c_{j k}^{T} l_{j k}\left(x_{j}^{W}, y_{j}^{W}\right)\right),
$$

where $c_{i}^{P}$ is the specific purchasing cost from the $i$ th supplier, $c_{j k}^{T}$ is the specific transportation cost between the $j$ th consignment store and $k$ th customer, and $l_{j k}$ is the length of the 

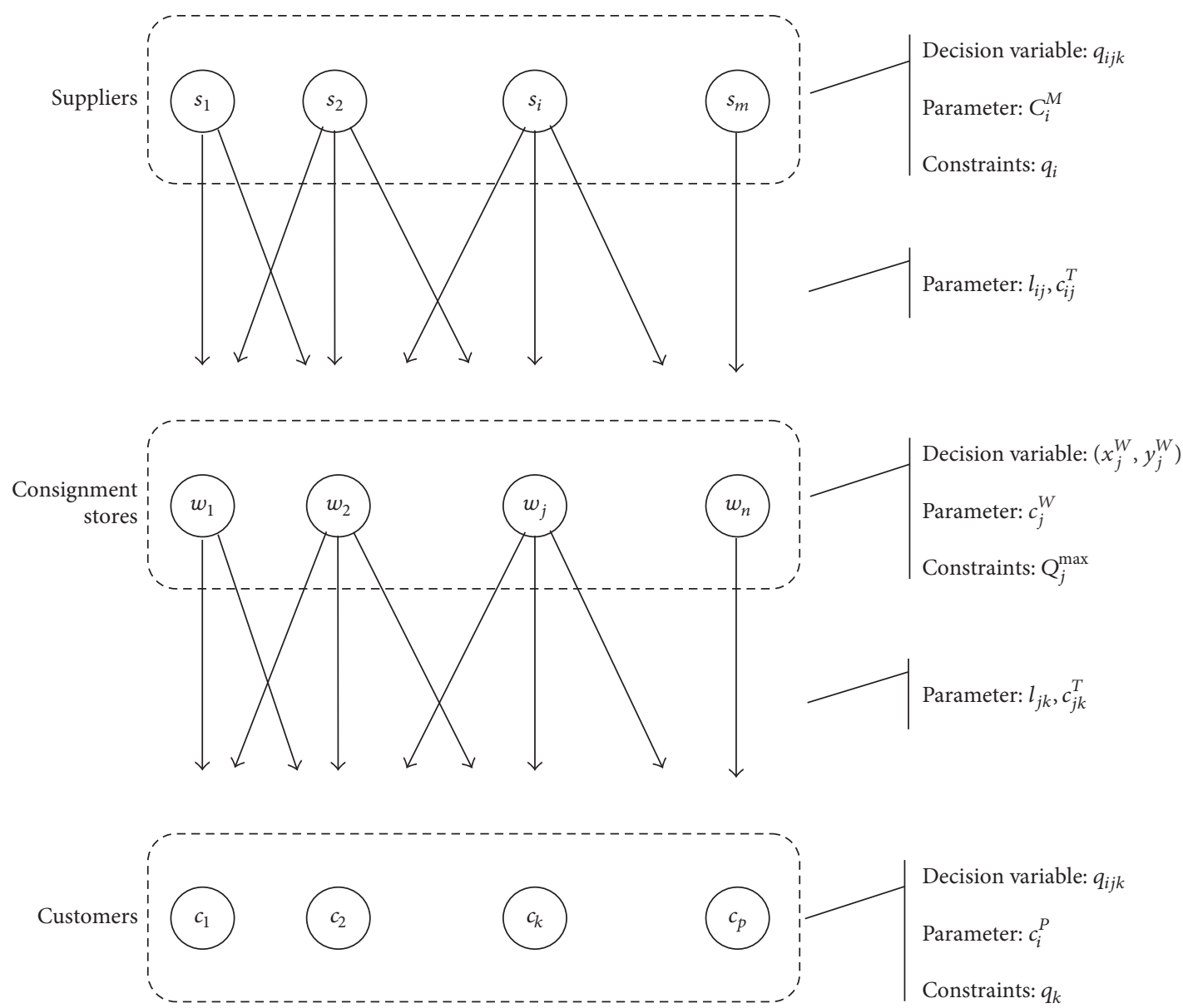

FIGURE 1: Model framework of consignment-store-based supply chain.

transportation route between the $j$ th consignment store and $k$ th customer.

The values of the products supplied, stored, and bought are limited by the following three constraints.

Constraint 1. Each time interval, no more than the capacity of the consignment store may be transported from suppliers to consignment stores (see the following equation):

$$
\sum_{i=1}^{m} \sum_{k=1}^{p} q_{i j k} \leq Q_{j}^{\max } \quad j \in(1,2, \ldots, n),
$$

where $Q_{j}^{\max }$ is the capacity of the $j$ th consignment store.

Constraint 2. All products manufactured by suppliers should be transported to consignment stores each time interval (see the following equation):

$$
\sum_{j=1}^{n} \sum_{k=1}^{p} q_{i j k}=q_{i} \quad i \in(1,2, \ldots, m),
$$

where $q_{i}$ is the total amount of product produced by the $i$ th supplier.
Constraint 3. Each time interval, amount of purchased products must reach the total demand of customers (see the following equation):

$$
\sum_{i=1}^{m} \sum_{j=1}^{n} q_{i j k}=q_{k} \quad k \in(1,2, \ldots, p),
$$

where $q_{k}$ is the total required amount of the $k$ th customer in the planning period.

The decision variables can only assume nonnegative values, so we associate sign restrictions with the abovementioned decision variables (see the following equation):

$$
q_{i j k}, x_{j}^{W}, y_{j}^{W} \geq 0 .
$$

\section{Black Hole Algorithm}

Black holes are places in the outer space where the gravitation force is so high that no particles even light can get out. Black holes are born when stars die. The environment of black holes can be analyzed, but the black holes are invisible. The Schwarzschild radius is the radius of the event horizon. If the distance between a particle (star, proton, electron, photon, etc.) is much higher than the Schwarzschild radius, then the particle can move in any direction. If this distance is larger 


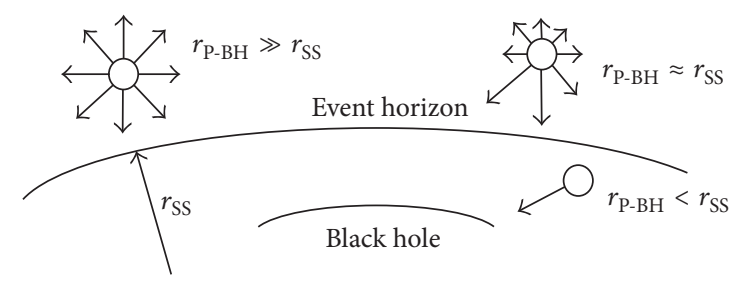

FIgURE 2: Behavior of particles depending on the distance from black hole.

than the Schwarzschild radius but this difference is not too much, the space-time is deformed, and more particles are moving towards the center of the black hole than in other directions. If a particle reaches the Schwarzschild radius, then it can move only towards the center of the black hole (Figure 2). The black hole optimization is based on this phenomenon of black holes.

The first phase of the black hole optimization is the so called big-bang, when a new generation of stars is generated in the search space. Each star represents one solution of the optimization problem. The coordinates of the $i$ th star in the $n$-dimensional search space represent the decision variables of the $n$-dimensional optimization problem.

$$
\vec{x}_{j}^{s_{i}}=\left(x_{1}^{S_{i}}, x_{2}^{S_{i}}, \ldots, x_{n}^{S_{i}}\right) .
$$

The second phase of the algorithm is the evaluation of the stars. The stars are evaluated with the value of the objective functions (gravity force represented by the star).

$$
f^{S_{i}}=f^{S_{i}}\left(x_{1}^{S_{i}}, x_{2}^{S_{i}}, \ldots, x_{n}^{S_{i}}\right) .
$$

The third phase is to choose one or more black holes. Black holes are the stars with the highest gravity force.

$$
f^{\mathrm{BH}}=\max _{i}\left(f^{S_{i}}\right) .
$$

The fourth phase of the algorithm is to move the stars towards the black holes in the search space. There are different operators to calculate the new locations of the stars. The basic operator uses only the gravity force of the black holes and the gravity force of stars is not taken into consideration. There are two main types of operators: the first type calculates the new position of the stars depending on the gravity forces among stars and black holes, and the second type does not take into account the gravity forces (see the following equation):

$$
x_{j}^{S_{i}}(t+\Delta t)=x_{j}^{S_{i}}(t)+\mathrm{Rnd} \cdot\left(x_{j}^{\mathrm{BH}}(t)-x_{j}^{S_{i}}(t)\right) .
$$

The movement of stars towards the black hole changes the decision variables of the solution represented by the moving star so that the decision variables will move to the decision variables of the best solution represented by the black hole (Figure 3).

Stars reaching the event horizon will be absorbed and a new star is generated in the search space. The radius of the event horizon (the Schwarzschild radius) is calculated as follows:

$$
R^{\mathrm{EH}}=\frac{f^{\mathrm{BH}}}{\sum_{i=1}^{n} f^{S_{i}}},
$$

where $R^{\mathrm{EH}}$ is the radius of event horizon, $f^{\mathrm{BH}}$ is the gravity force of the black hole, and $f^{S_{i}}$ is the gravity force of the $i$ th star.

The fifth phase is the evaluation of stars. Stars with the best gravity force will be the new black holes, and the old black holes become stars. This role of this fifth phase is the same as the role of the mutation operator of genetic algorithms: to avoid the local optimum. Termination criteria of the algorithm can be the number of iteration steps, computational time, or the measure of convergence.

If the location of the optimum is inside the event horizon, it is impossible to find it, because all stars inside the event horizon are absorbed. Stephen Hawking published a theoretical argument for the existence of blackbody radiation [41]. Virtual particle-antiparticle pairs, like photons or neutrinos, are being created near the event horizon of the black hole. These particle-antiparticle pairs annihilate each other or one of them falls into the black hole and the other one escapes as Hawking radiation due to quantum effects. The black hole loses a part of its energy and its mass. This Hawking radiation is called black hole evaporation. It is possible to apply this black hole evaporation to search inside the event horizon [42]. This application means that a little change occurs in the location of the black hole, so that a small part of the old event horizon is available for the stars.

$$
x_{j}^{\mathrm{BH}}(t+\Delta t)=x_{j}^{\mathrm{BH}}(t)+\varepsilon, \quad|\varepsilon| \ll R^{\mathrm{EH}} .
$$

Another way to open the event horizon of black holes for the stars to search for the best solution is to decrease the measure of event horizon. The pseudocode shows the developed approach (Pseudocode 1).

The described Pseudocode 1 makes it possible to replicate the implementation. It is also possible to have more than one black hole; in this case, the movement of stars towards the black holes is similar to the gravity search algorithm [43].

\section{Sensitivity Analysis}

Within the frame of this chapter, the sensitivity analysis of the black hole algorithm is described. The following 10 different benchmark functions were used to evaluate the above-described black hole algorithm:

(i) The $n$-dimensional nonconvex Shifted sphere function is evaluated on the hypercube $x_{i} \in[-100,100]$. It has a global minimum at $f_{1}\left(s_{1}, \ldots, s_{i}, \ldots, s_{d}\right)=b$.

(ii) The $n$-dimensional shifted Schwefel function is evaluated on the hypercube $x_{i} \in[-100,100]$. It has a global minimum at $f_{2}\left(s_{1}, \ldots, s_{i}, \ldots, s_{d}\right)=b$.

(iii) The $n$-dimensional shifted elliptic function is evaluated on the hypercube $x_{i} \in[-100,100]$. It has a global minimum at $f_{3}\left(s_{1}, \ldots, s_{i}, \ldots, s_{d}\right)=b$. 

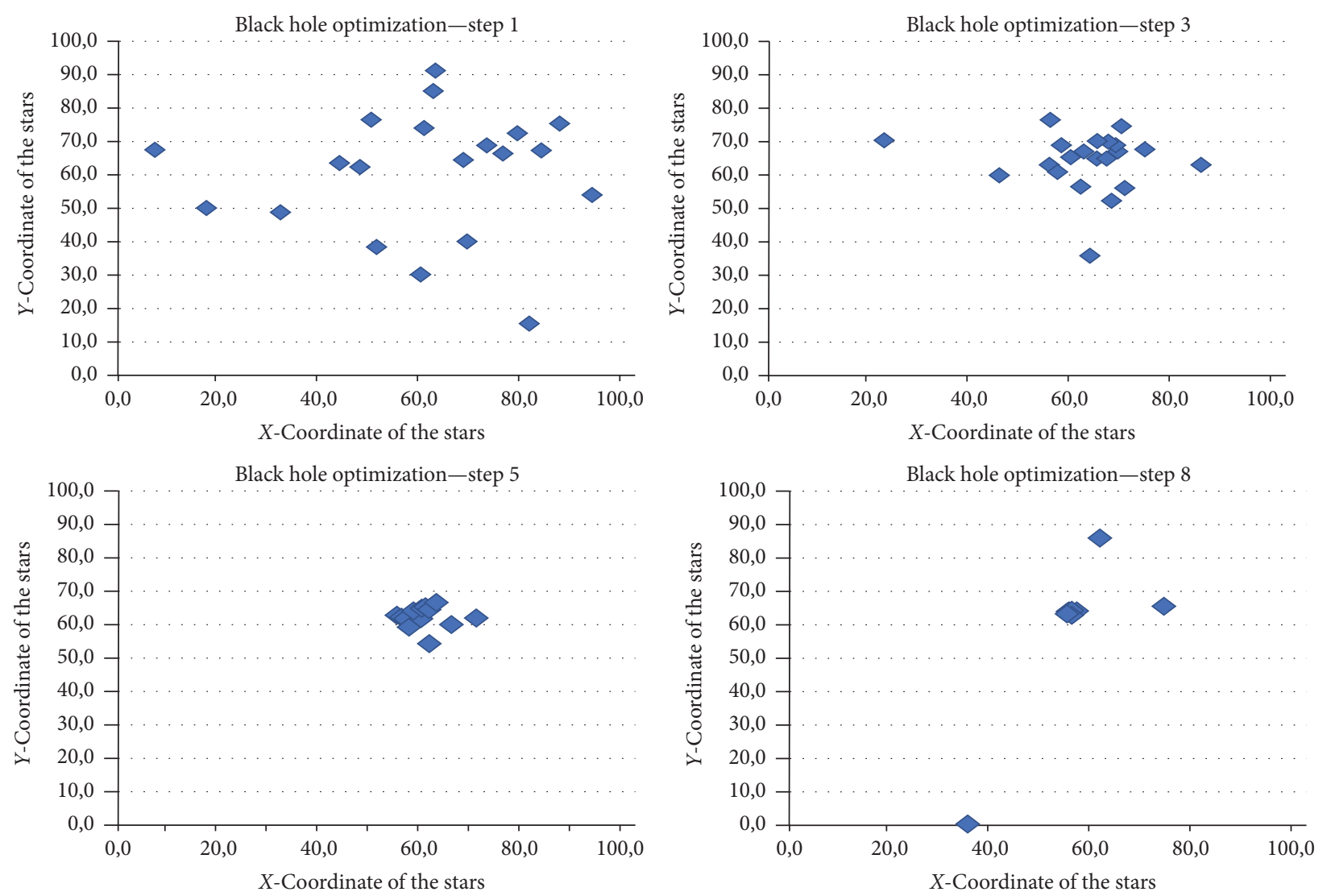

FIGURE 3: Moving of stars in BHO (step 1: initialization of stars; step 3: stars are moving in the direction of the black hole; step 5: stars are nearing the event horizon; step 8: some stars cross the Schwarzschild radius, they are absorbed, and new stars are generated in the search space).

Input: number of stars, objective function, constraints, sign restrictions, termination criteria Output: optimal solution

// Initialization

(1) generate feasible solutions randomly in the $n$-dimensional search space (9)

// Pre-evaluation

(2) for each stars, evaluate the objective function ((2)-(4), (10))

// Loop until the termination criteria satisfy

While (termination criteria satisfy) do

// Selection of the black hole

(3) select the best star that has the best value to become a black hole (11)

// Hawking radiation

(4) change the position of the black hole (14)

// movement of stars towards the black hole

(5) move the stars towards the black holes (12) while constraints ((5)-(8)) are taken into consideration

// Check the position of stars

(6) if star is inside the Schwarzschild radius

absorb the star and generate a new one in the search space (13) end if

// Evaluation

(7) for each stars, evaluate the objective function ((2)-(4), (10))

End of while

Pseudocode 1: Pseudocode of BHA. 
TABLE 2: Error values of BHO in the case of 10 benchmark functions after 100 iteration steps.

\begin{tabular}{|c|c|c|c|c|}
\hline Evaluation function & Standard $\mathrm{BHO}$ & $\begin{array}{l}\text { BHO with } \\
\text { moving black } \\
\text { hole location }\end{array}$ & $\begin{array}{l}\text { BHO with } \\
\text { decreasing event } \\
\text { horizon }\end{array}$ & $\begin{array}{l}\text { Complex } \\
\mathrm{BHO}\end{array}$ \\
\hline \multicolumn{5}{|l|}{ Shifted sphere function } \\
\hline$f_{1}\left(x_{1}, \ldots, x_{i}, \ldots, x_{d}\right)=\sum_{i=1}^{d}\left(x_{i}-s_{i}\right)^{2}+b$ & $1,02 E-7$ & $1,02 E-7$ & $9,55 E-8$ & $4,76 E-8$ \\
\hline $\begin{array}{l}\text { Shifted Schwefel function } \\
f_{2}\left(x_{1}, \ldots, x_{i}, \ldots, x_{d}\right)=\sum_{i=1}^{d}\left(\sum_{j=1}^{i}\left(x_{i}-s_{i}\right)\right)^{2}+b\end{array}$ & $8,82 E-6$ & $8,34 E-6$ & $7,21 E-6$ & $4,02 E-6$ \\
\hline $\begin{array}{l}\text { Shifted elliptic function } \\
f_{3}\left(x_{1}, \ldots, x_{i}, \ldots, x_{d}\right)=\sum_{i=1}^{d}\left(10^{6}\right)^{(i-1) /(d-1)}\left(x_{i}-s_{i}\right)^{2}+b\end{array}$ & $4,32 E-7$ & $4,12 E-7$ & $2,62 E-7$ & $1,92 E-7$ \\
\hline $\begin{array}{l}\text { Styblinski-Tang function } \\
f_{4}\left(x_{1}, \ldots, x_{i}, \ldots, x_{d}\right)=\frac{1}{2} \sum_{i=1}^{d}\left(x_{i}^{4}-16 x_{i}^{2}+5 x_{i}\right)\end{array}$ & $2,91 E-6$ & $1,38 E-6$ & $6,12 E-7$ & $4,65 E-7$ \\
\hline $\begin{array}{l}\text { Rosenbrock function } \\
f_{5}\left(x_{1}, \ldots, x_{i}, \ldots, x_{d}\right)=\sum_{i=1}^{d-1}\left[100\left(x_{i+1}-x_{i}^{2}\right)^{2}+\left(x_{i}-1\right)^{2}\right]\end{array}$ & $5,50 E-6$ & $5,44 E-6$ & $2,92 E-6$ & $2,02 E-6$ \\
\hline $\begin{array}{l}\text { Rastrigin function } \\
f_{6}\left(x_{1}, \ldots, x_{i}, \ldots, x_{d}\right)=10+\sum_{i=1}^{d}\left[x_{i}^{2}-10 \cos 2 \pi x_{i}\right]\end{array}$ & $2,45 E-6$ & $2,32 E-6$ & $1,07 E-6$ & $9,69 E-7$ \\
\hline $\begin{array}{l}\text { Ackley function } \\
f_{7}(x, y)=-20 \cdot e^{-0.2 \sqrt{0.5\left(x^{2}+y^{2}\right)}}-e^{(0.5(\cos 2 \pi x)+\cos 2 \pi y)}+e+20\end{array}$ & $1,14 E-7$ & $1,01 E-7$ & $9,63 E-8$ & $7,17 E-8$ \\
\hline $\begin{array}{l}\text { Beale function } \\
f_{8}(x, y)=(1.5-x+x y)^{2}+\left(2.25-x+x y^{2}\right)^{2}+\left(2.625-x+x y^{3}\right)^{2}\end{array}$ & $2,29 E-7$ & $2,26 E-7$ & $1,91 E-7$ & $1,21 E-7$ \\
\hline $\begin{array}{l}\text { Booth function } \\
f_{9}(x, y)=(x+2 y-7)^{2}+(2 x+y-5)^{2}\end{array}$ & $5,31 E-6$ & $2,11 E-6$ & $5,51 E-7$ & $3,26 E-7$ \\
\hline $\begin{array}{l}\text { Goldstein-Price function } \\
f_{10}(x, y)=\left[1+(x+y+1)^{2}\left(19-14 x+3 x^{2}-14 y+6 x y+3 y^{2}\right)\right][30+ \\
\left.(2 x-3 y)^{2}\left(18-32 x+12 x^{2}+48 y-36 x y+27 y^{2}\right)\right]\end{array}$ & $3,34 E-8$ & $8,22 E-9$ & $6,31 E-9$ & $3,12 E-9$ \\
\hline
\end{tabular}

(iv) The $n$-dimensional Styblinski-Tang function is evaluated on the hypercube $x_{i} \in[-5,5]$. It has a global minimum at $f_{4}(-2.903534, \ldots,-2.903534)=$ -39.16599 .

(v) The $n$-dimensional Rosenbrock function is evaluated on the hypercube $x_{i} \in[-\infty, \infty]$. It has a global minimum at $f_{5}(1, \ldots, 1, \ldots, 1)=0$.

(vi) The $n$-dimensional Rastrigin function is evaluated on the hypercube $x_{i} \in[-5.12,5.12]$. It has a global minimum at $f_{6}(0, \ldots, 0, \ldots, 0)=0$.

(vii) The 2-dimensional Ackley function is evaluated on the $x_{i} \in[-5,5]$ square. It has a global minimum at $f_{7}(0,0)=0$.

(viii) The 2-dimensional Beale function is evaluated on the $x_{i} \in[-4.5,4.5]$ square. It has a global minimum at $f_{8}(3,0.5)=0$.

(ix) The 2-dimensional Booth function is evaluated on the $x_{i} \in[-10,10]$ square. It has a global minimum at $f_{9}(1,3)=0$. (x) The 2-dimensional Goldstein-Price function is evaluated on the $x_{i} \in[-2,2]$ square. It has a global minimum at $f_{10}(0,-1)=3$.

The aim of this evaluation is to analyze the effect of permanently decreased Schwarzschild radius and the changes occurred in the location of black holes.

As Table 2 demonstrates, the permanently decreased Schwarzschild radius and the moving black hole effect decreased the error value after 100 iteration steps. The problem size was fixed as a 10-dimensional problem in the case of $f_{1}$ to $f_{6}$. The efficiency of the algorithm in the case of different problem sizes (different search space dimensions) is demonstrated in the next chapter. The average error value was reduced by $36 \%$ with a deviation of $19 \%$ (Table 3 ).

In order to demonstrate how black hole implementation performs when problem size increases, we tested the algorithm both with test functions and with the consignmentstore-based supply chain problem.

As Table 4 shows, the increased size of the problem led to the increase of the required iteration steps to reach the predefined error value that is based on the performance of 


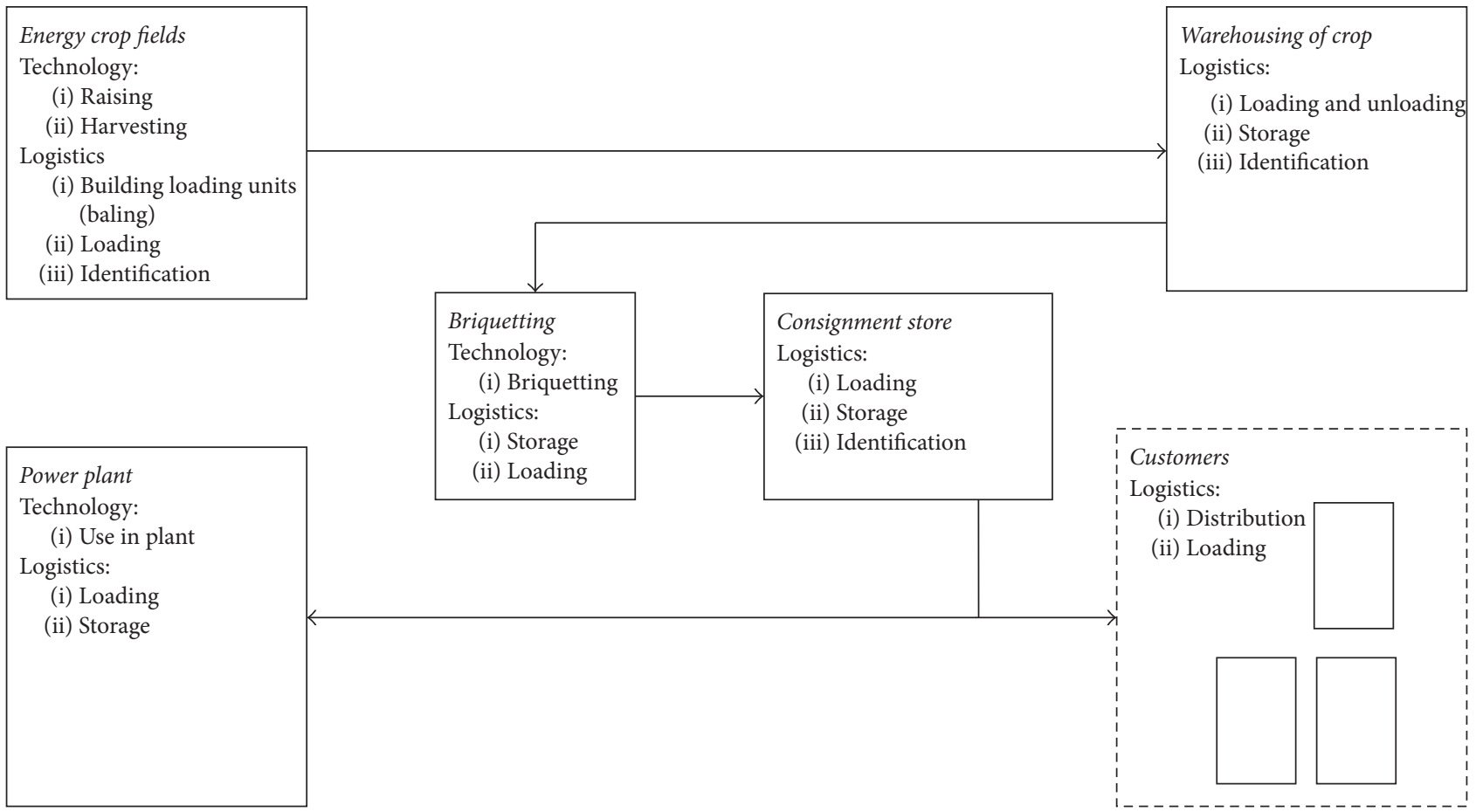

FIgURE 4: Complex power plant supply chain.

TABLE 3: Error value decline using moving black holes and permanently decreasing Schwarzschild radius.

\begin{tabular}{lc}
\hline Evaluation function & Error value decline \\
\hline Shifted sphere function & $47 \%$ \\
Shifted Schwefel function & $46 \%$ \\
Shifted elliptic function & $44 \%$ \\
Styblinski-Tang function & $16 \%$ \\
Rosenbrock function & $37 \%$ \\
Rastrigin function & $40 \%$ \\
Ackley function & $63 \%$ \\
Beale function & $53 \%$ \\
Booth function & $6 \%$ \\
Goldstein-Price function & $9 \%$ \\
\hline
\end{tabular}

the algorithm in the case of a 10-dimensional search space (Table 2). The highest iteration step was required in the case of the consignment-store-based supply chain problem because of the specific constraints and sign restrictions.

\section{Numerical Analysis of Consignment-Store- Based Supply Chain}

Within the frame of this chapter, a case study will be analyzed. The aim of this chapter is to analyze the design of an energy crop supply chain of biomass-fired power plants, especially from the point of view of integrated facility location. The model shown in Figure 4 includes the whole energy crop supply chain from harvesting crop in the crop fields through briquetting plants to the distribution for power plants and customers through consignment stores. The following parameters are taken into consideration: the total amount of harvested energy crop, required briquette amount by each power plant and the cluster of customers, transportation distances, specific transportation costs, type of transportation devices (average truck capacity), and location of crop fields, power plants, and customers. The objective function is a cost function based on the transportation processes from energy crop fields to briquetting plants and from briquetting plants to power plants and communal customers. The decision variables are the following: (a) how many energy crops from crop field through consignment stores to customers and power plants should be transported; (b) location of each consignment store.

Figure 5 demonstrates the results of the optimization of the above-mentioned complex supply chain. The datasets represent simple specification of the system so that the results of the optimization can be checked.

In the first case, customers and power plants need the same quantity of briquette; therefore the central position of both consignment stores is correct. The first power plant and the first and second customer's clusters are assigned to the first consignment store, while the second power plant and the third and the fourth customer's clusters are assigned to the second consignment store.

In the second case, the third customer's cluster has a greater demand of briquette. In this case, the second consignment store's location is not centralized in order to minimize the materials handling costs of the whole supply chain. 
TABLE 4: Number of required iteration steps to reach the predefined error value (PEV).

\begin{tabular}{|c|c|c|c|}
\hline \multirow{2}{*}{ Evaluation function } & \multicolumn{3}{|c|}{ Problem size } \\
\hline & $d=5$ & $d=10$ & $d=25$ \\
\hline \multicolumn{4}{|l|}{ Shifted sphere function } \\
\hline$f_{1}\left(x_{1}, \ldots, x_{i}, \ldots, x_{d}\right)=\sum_{i=1}^{d}\left(x_{i}-s_{i}\right)^{2}+b$ & 94 & 100 & 122 \\
\hline \multicolumn{4}{|l|}{$\mathrm{PEV}=4,76 E-8$} \\
\hline \multicolumn{4}{|l|}{ Shifted Schwefel function } \\
\hline$f_{2}\left(x_{1}, \ldots, x_{i}, \ldots, x_{d}\right)=\sum_{i=1}^{a}\left(\sum_{i=1}^{i}\left(x_{i}-s_{i}\right)\right)^{2}+b$ & 92 & 100 & 134 \\
\hline \multicolumn{4}{|l|}{$\mathrm{PEV}=4,02 E-6$} \\
\hline \multicolumn{4}{|l|}{ Shifted elliptic function } \\
\hline$f_{3}\left(x_{1}, \ldots, x_{i}, \ldots, x_{d}\right)=\sum_{i=1}^{a}\left(10^{6}\right)^{(i-1) /(d-1)}\left(x_{i}-s_{i}\right)^{2}+b$ & 81 & 100 & 156 \\
\hline \multicolumn{4}{|l|}{$\mathrm{PEV}=1,92 E-7$} \\
\hline \multicolumn{4}{|l|}{ Styblinski-Tang function } \\
\hline$f_{4}\left(x_{1}, \ldots, x_{i}, \ldots, x_{d}\right)=\frac{1}{2} \sum_{i=1}^{d}\left(x_{i}^{4}-16 x_{i}^{2}+5 x_{i}\right)$ & 85 & 100 & 162 \\
\hline \multicolumn{4}{|l|}{$\mathrm{PEV}=4,65 E-7$} \\
\hline \multicolumn{4}{|l|}{ Rosenbrock function } \\
\hline$f_{5}\left(x_{1}, \ldots, x_{i}, \ldots, x_{d}\right)=\sum^{a-1}\left[100\left(x_{i+1}-x_{i}^{2}\right)^{2}+\left(x_{i}-1\right)^{2}\right]$ & 93 & 100 & 133 \\
\hline \multicolumn{4}{|l|}{$\mathrm{PEV}=2,02 E-6$} \\
\hline \multicolumn{4}{|l|}{ Rastrigin function } \\
\hline$f_{6}\left(x_{1}, \ldots, x_{i}, \ldots, x_{d}\right)=10+\sum_{i=1}^{a}\left[x_{i}^{2}-10 \cos 2 \pi x_{i}\right]$ & 84 & 100 & 144 \\
\hline \multicolumn{4}{|l|}{$\mathrm{PEV}=9,69 E-7$} \\
\hline $\begin{array}{l}\text { Consignment-store-based supply chain problem } \\
\mathrm{PEV}=5,63 E-7\end{array}$ & 89 & 100 & 168 \\
\hline
\end{tabular}
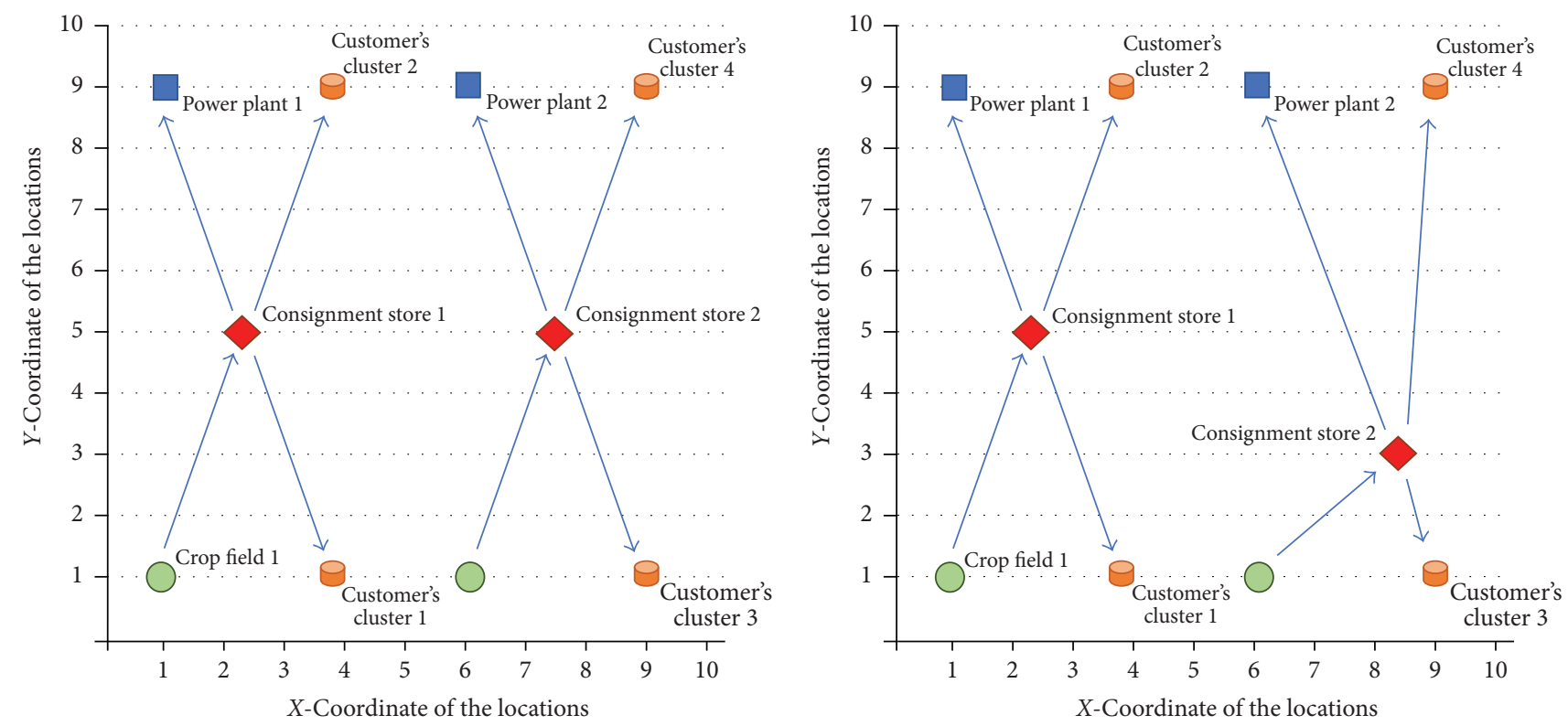

FIGURE 5: Results of the facility location and assignment problem of energy crop supply chain. 
The optimization of this complex supply chain problem can lead to the decrease of different costs, like transportation costs, warehousing costs, and materials handling costs (packaging, loading, unloading, and building of loading units).

\section{Conclusions and Further Research Directions}

This study developed a methodological approach for design of consignment-store-based supply chains. In this paper, firstly, we reviewed and systematically categorized the recent works presented for consignment-store-based supply chain optimization. Then, motivated by the gaps in the literature, a model for companies performing their purchasing through consignment stores is developed. Two models were proposed: the framework model shows the levels of supply chain, while the second model as a case study focuses on power plant supply. The integrated model included facility location and assignment problems, which were solved with black hole optimization algorithm. The sensitivity analysis showed the efficiency of two advanced $\mathrm{BHO}$ operators and a numerical example shows the efficiency of the algorithm.

The scientific contributions of this paper are the following: integrated model for consignment-store-based supply chain, black-hole-optimization-based heuristic algorithm with enhanced convergence through integration of phenomena of real black holes, like dynamic black hole location, and decreased event horizon. The results can be generalized, because the model can be applied for in-plant supply, especially in the case of milk-run-based just-in-sequence supply. The described methods make it possible to support managerial decisions; the operation strategy of the supply chain and the consignment contract can be influenced by the results of the above-described contribution.

However, there are also directions for further research. First, although the transportation routes as distances among the locations are considered in this paper, the capacities of vehicles are not taken into consideration. In further studies, the model can be extended to a more complex model including capacities of vehicles and store capacities of locations. Second, this study only considered the black hole optimization method as possible solution algorithm for the described NP-hard problem. In reality, other heuristic methods can be also suitable for the solution of the problem.

Third, the convergence of the described algorithm can be improved using other operators and the behavior of $\mathrm{BHO}$ to other optimization approaches can be tested. However, there is a great body of research dealing with testing of performance of different metaheuristic optimization methods, especially from the point of view of "novel" algorithm, but these tests are sometimes inconsistent. This inconsistency can be caused by the optimization behavior. For example, the comparison of black hole algorithm and particle swarm optimization showed that the performance of $\mathrm{BHO}$ is poorer than the performance of PSO [44], while the test in another source showed that the performance of $\mathrm{BHO}$ is better than the performance of genetic algorithm or PSO [45]. This should be also considered in the future research.

\section{Conflicts of Interest}

The authors declare that there are no conflicts of interest regarding the publication of this paper.

\section{Acknowledgments}

This project has received funding from the European Union's Horizon 2020 research and innovation programme under Grant Agreement no. 691942. This research was partially carried out within the framework of the Center of Excellence of Mechatronics and Logistics at the University of Miskolc.

\section{References}

[1] J. Hallikas and K. Lintukangas, "Purchasing and supply: an investigation of risk management performance," International Journal of Production Economics, vol. 171, pp. 487-494, 2016.

[2] A. Matopoulos, J. E. Bell, and E. Aktas, "The use of modelling in purchasing/supply management research," Journal of Purchasing and Supply Management, vol. 22, no. 4, pp. 262-265, 2016.

[3] M. Immonen, J. Hallikas, and M. Pynnönen, "Antecedents of system purchasing in B2B services," Journal of Purchasing and Supply Management, vol. 22, no. 3, pp. 205-213, 2016.

[4] Q. Zhang, A. Sundaramoorthy, I. E. Grossmann, and J. M. Pinto, "Multiscale production routing in multicommodity supply chains with complex production facilities," Computers \& Operations Research, vol. 79, pp. 207-222, 2017.

[5] K. Govindan and H. Soleimani, "A review of reverse logistics and closed-loop supply chains: a journal of cleaner production focus," Journal of Cleaner Production, vol. 142, no. 1, pp. 371-384, 2017.

[6] Y. Ma, F. Yan, K. Kang, and X. Wei, "A novel integrated production-distribution planning model with conflict and coordination in a supply chain network," Knowledge-Based Systems, vol. 105, pp. 119-133, 2016.

[7] A. Diabat and R. Deskoores, "A hybrid genetic algorithm based heuristic for an integrated supply chain problem," Journal of Manufacturing Systems, vol. 38, pp. 172-180, 2016.

[8] M. S. Pishvaee and M. Rabbani, "A graph theoretic-based heuristic algorithm for responsive supply chain network design with direct and indirect shipment," Advances in Engineering Software, vol. 42, no. 3, pp. 57-63, 2011.

[9] S.-C. Liu and J.-R. Chen, "A heuristic method for the inventory routing and pricing problem in a supply chain," Expert Systems with Applications, vol. 38, no. 3, pp. 1447-1456, 2011.

[10] H. Chávez, K. K. Castillo-Villar, L. Herrera, and A. Bustos, "Simulation-based multi-objective model for supply chains with disruptions in transportation," Robotics and ComputerIntegrated Manufacturing, vol. 43, pp. 39-49, 2017.

[11] M. Dorigatti, A. Guarnaschelli, O. Chiotti, and H. E. Salomone, "A service-oriented framework for agent-based simulations of collaborative supply chains," Computers in Industry, vol. 83, pp. 92-107, 2016.

[12] H. Ge, J. Nolan, R. Gray, S. Goetz, and Y. Han, "Supply chain complexity and risk mitigation - A hybrid optimization-simulation model," International Journal of Production Economics, vol. 179, pp. 228-238, 2016.

[13] S. Ben Othman, H. Zgaya, M. Dotoli, and S. Hammadi, "An agent-based Decision Support System for resources' scheduling 
in Emergency Supply Chains," Control Engineering Practice, vol. 59, pp. 27-43, 2017.

[14] B. Dey, B. Bairagi, B. Sarkar, and S. K. Sanyal, "Group heterogeneity in multi member decision making model with an application to warehouse location selection in a supply chain," Computers Industrial Engineering, vol. 105, pp. 101-122, 2017.

[15] S. K. Zahran, M. Y. Jaber, and S. Zanoni, "The consignment stock case for a vendor and a buyer with delay-in-payments," Computers Industrial Engineering, vol. 98, pp. 333-349, 2016.

[16] S. C. Hackett, "Consignment contracting," Journal of Economic Behavior \& Organization, vol. 20, no. 2, pp. 247-253, 1993.

[17] S. Bylka, "Non-cooperative consignment stock strategies for management in supply chain," International Journal of Production Economics, vol. 143, no. 2, pp. 424-433, 2013.

[18] E. Bazan, M. Y. Jaber, S. Zanoni, and L. E. Zavanella, "Vendor Managed Inventory (VMI) with Consignment Stock (CS) agreement for a two-level supply chain with an imperfect production process with/without restoration interruptions," International Journal of Production Economics, vol. 157, no. 1, pp. 289-301, 2014.

[19] T. Li, S. P. Sethi, and J. Zhang, "Supply diversification with isoelastic demand," International Journal of Production Economics, vol. 157, no. 1, pp. 2-6, 2014.

[20] R. Batarfi, M. Y. Jaber, and S. Zanoni, "Dual-channel supply chain: a strategy to maximize profit," Applied Mathematical Modelling. Simulation and Computation for Engineering and Environmental Systems, vol. 40, no. 21-22, pp. 9454-9473, 2016.

[21] J. Ru and Y. Wang, "Consignment contracting: who should control inventory in the supply chain?" European Journal of Operational Research, vol. 201, no. 3, pp. 760-769, 2010.

[22] C. Fraser, "Schwarzschield radius," https://www.universetoday .com/39861/schwarzschild-radius/, 2017.

[23] A. P. Piotrowski, J. J. Napiorkowski, and P. M. Rowinski, "How novel is the "novel" black hole optimization approach?" Information Sciences, vol. 267, pp. 191-200, 2014.

[24] M. Dorigo and L. M. Gambardella, "Ant colonies for the travelling salesman problem," BioSystems, vol. 43, no. 2, pp. 7381, 1997.

[25] X.-S. Yang, Nature-Inspired Optimization Algorithms, Elsevier Inc., Amsterdam, Netherlands, 2014.

[26] V. Bhargava, S. E. K. Fateen, and A. Bonilla-Petriciolet, "Cuckoo Search: A new nature-inspired optimization method for phase equilibrium calculations," Fluid Phase Equilibria, vol. 337, pp. 191-200, 2013.

[27] M. Lozano, C. García-Martínez, F. J. Rodríguez, and H. M. Trujillo, "Optimizing network attacks by artificial bee colony," Information Sciences, vol. 377, pp. 30-50, 2017.

[28] T. Niknam, S. Sharifinia, and R. Azizipanah-Abarghooee, "A new enhanced bat-inspired algorithm for finding linear supply function equilibrium of GENCOs in the competitive electricity market," Energy Conversion and Management, vol. 76, pp. 10151028, 2013.

[29] A. R. McKendall Jr., J. Shang, and S. Kuppusamy, "Simulated annealing heuristics for the dynamic facility layout problem," Computers \& Operations Research, vol. 33, no. 8, pp. 2431-2444, 2006.

[30] S. K. Saha, R. Kar, D. Mandal, and S. P. Ghoshal, "Gravitation search algorithm: Application to the optimal IIR filter design," Journal of King Saud University_Engineering Sciences, vol. 26, no. 1, pp. 69-81, 2014.
[31] P. R. Srivastava, "A cooperative approach to optimize the Printed Circuit Boards drill routing process using Intelligent Water Drops," Computers \& Electrical Engineering, vol. 43, pp. 270277, 2015.

[32] T. Bányai, P. Veres, and B. Illés, "Heuristic supply chain optimization of networked maintenance companies," in Proceedings of the 25th DAAAM International Symposium on Intelligent Manufacturing and Automation, DAAAM 2014, pp. 46-55, Vienna, Austria, 2015.

[33] J. Zhang, K. Liu, Y. Tan, and X. He, "Random black hole particle swarm optimization and its application," in Proceedings of the 2008 IEEE International Conference Neural Networks and Signal Processing, ICNNSP, pp. 359-365, Nanjing, China, June 2008.

[34] S. Yaghoobi and H. Mojallali, "Modified Black Hole Algorithm with Genetic Operators," International Journal of Computational Intelligence Systems, vol. 9, no. 4, pp. 652-665, 2016.

[35] T. Wang, W. F. Liu, and C. F. Liu, "Optimization algorithm of black-hole based on Euclidean distance," Journal of Shenyang University of Technology, vol. 38, no. 2, pp. 201-205, 2016.

[36] H. R. E. H. Bouchekara, "Optimal design of electromagnetic devices using a black-Hole-Based optimization technique," IEEE Transactions on Magnetics, vol. 49, no. 12, pp. 5709-5714, 2013.

[37] T. Wang, X. W. Gao, and Z. J. Jiang, "Parameters optimizing of LSSVM based on Black hole algorithm," Journal of Northeastern University, vol. 35, no. 2, pp. 170-174, 2014.

[38] A. Hatamlou, "Black hole: a new heuristic optimization approach for data clustering," Information Sciences. An International Journal, vol. 222, pp. 175-184, 2013.

[39] R. Azizipanah-Abarghooee, T. Niknam, F. Bavafa, and M. Zare, "Short-term scheduling of thermal power systems using hybrid gradient based modified teaching-learning optimizer with black hole algorithm," Electric Power Systems Research, vol. 108, pp. 16-34, 2014.

[40] H. R. E. H. Bouchekara, "Optimal power flow using black-holebased optimization approach," Applied Soft Computing Journal, vol. 24, pp. 879-888, 2014.

[41] S. W. Hawking, "Particle creation by black holes," Communications in Mathematical Physics, vol. 43, no. 3, pp. 199-220, 1975.

[42] M. Nemati and H. Momeni, "Black holes algorithm with fuzzy Hawking radiation," International Journal of Scientific \& Technology Research, vol. 3, no. 6, pp. 85-88, 2014.

[43] O. Bozorg-Haddad, M. Janbaz, and H. A. Loáiciga, "Application of the gravity search algorithm to multi-reservoir operation optimization," Advances in Water Resources, vol. 98, pp. 173-185, 2016.

[44] H. Gupta, A. Gupta, S. K. Gupta, P. Nayak, and T. Shrivastava, "How effective is black hole algorithm?" in Proceedings of the 2016 2nd International Conference on Contemporary Computing and Informatics (IC3I), pp. 474-478, Greater Noida, India, December 2016.

[45] M. Farahmandian and A. Hatamlou, "Solving optimization problems using black hole algorithm," Journal of Advanced Computer Science \& Technology, vol. 4, pp. 68-74, 2015. 


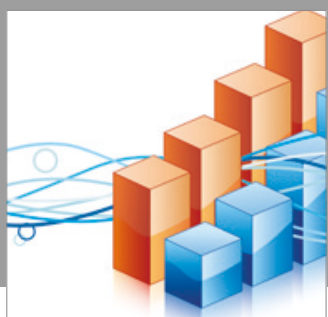

Advances in

Operations Research

vatersals

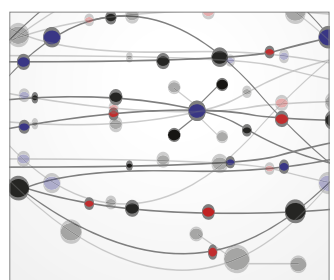

\section{The Scientific} World Journal
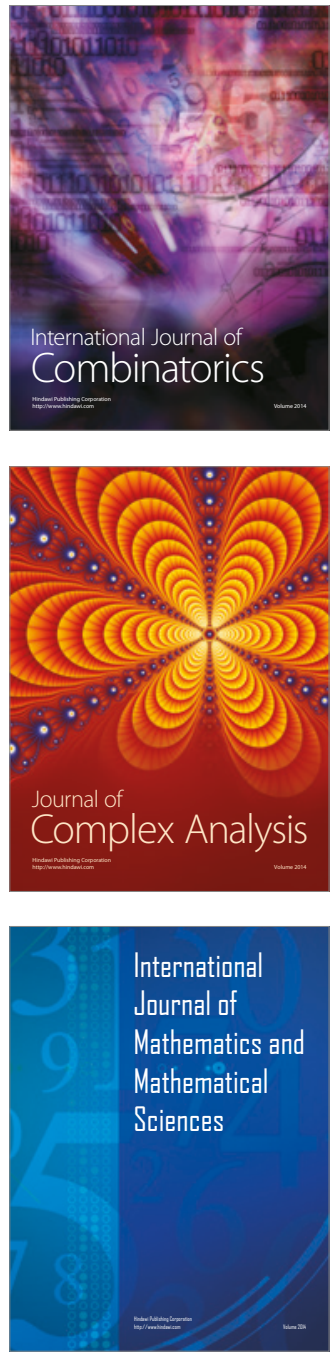
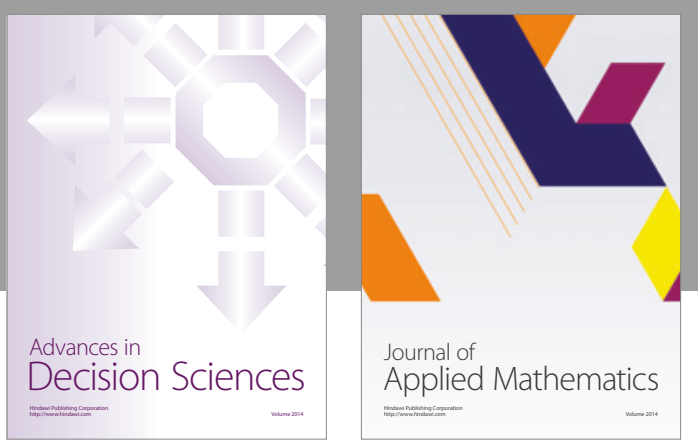

Algebra

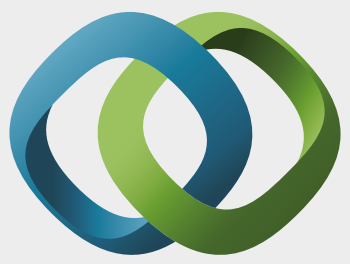

\section{Hindawi}

Submit your manuscripts at

https://www.hindawi.com
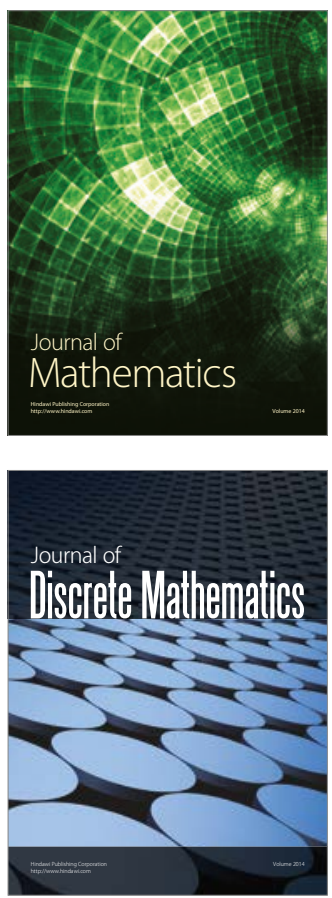

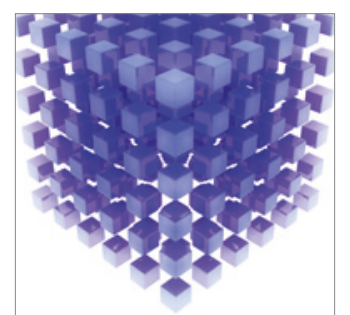

Mathematical Problems in Engineering
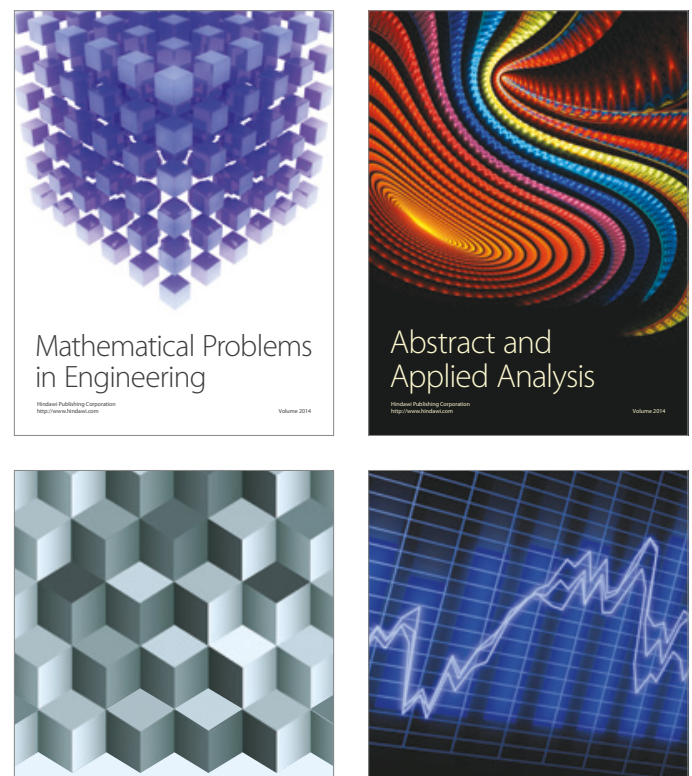

Journal of

Function Spaces

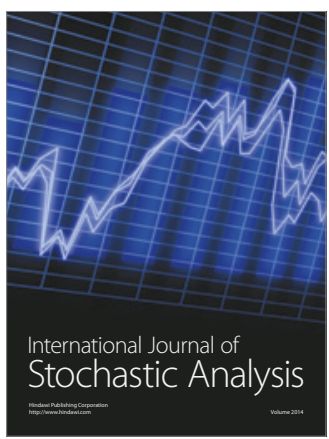

Probability and Statistics
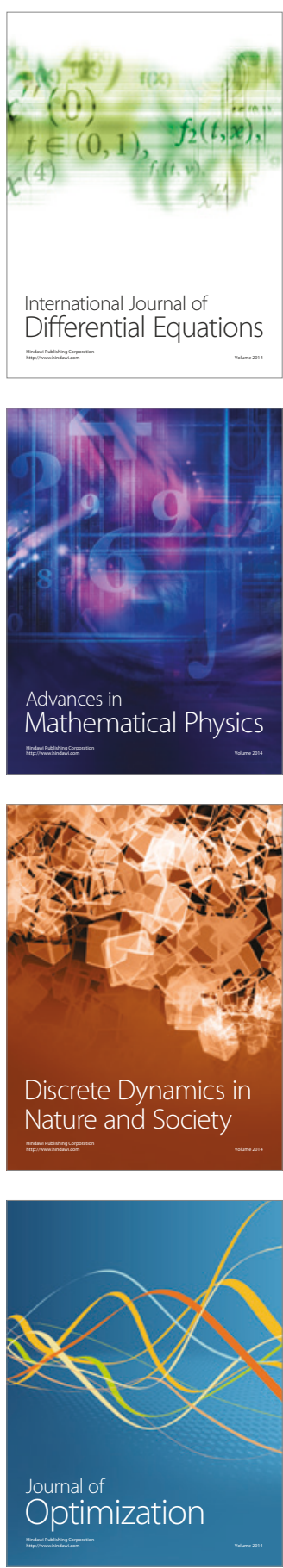